\title{
La educación virtual en el pensamiento crítico de los estudiantes universitarios
}

\section{Virtual education in the critical thinking of university students}

\author{
Carlos Arturo Valencia Morocho ${ }^{1}$ \\ Universidad César Vallejo \\ cvalencia@cientifica.edu.pe \\ ORCID: https://orcid.org/0000-0003-1515-1760
}

Citar como: Valencia, C. (2021). La educación virtual en el pensamiento crítico de los estudiantes universitarios. Desde el Sur, 13(2), e0018

\begin{abstract}
RESUMEN
El objetivo fue determinar la influencia que genera la educación virtual en el pensamiento crítico en los estudiantes durante la pandemia. Corresponde al paradigma positivista, tipo aplicada, nivel explicativo, enfoque cuantitativo, método hipotético-deductivo, diseño cuasiexperimental. La muestra fue no probabilística y estuvo conformada por 68 estudiantes, divididos en el grupo experimental con 35 estudiantes y el grupo control con 33 estudiantes. A ambos se les aplicó un pretest con una prueba de 20 ítems con textos argumentativos para evaluar el pensamiento crítico. Al grupo control se le aplicó en modalidad virtual con la enseñanza tradicional y al grupo experimental, el programa de enseñanza virtual a través del foro de discusión. Prueba similar se aplicó a ambos grupos en el postest. Después de la aplicación del programa, son significativos los resultados obtenidos por medio de la prueba de la $U$ de Mann Whitney $(81,00, Z=-6,142<, 05)$. En conclusión, la educación virtual influye significativamente en el pensamiento crítico.
\end{abstract}

1 Doctor en Educación y magíster en Docencia y Gestión Educativa por la Universidad César Vallejo. Asimismo, es licenciado en Educación, especialidad Lenguaje, y bachiller en Lingüística por la Universidad Nacional Mayor de San Marcos. Se desempeña como docente de Cursos Básicos de la Universidad Científica del Sur y en otras universidades como docente de Formación Humanística. 


\section{PALABRAS CLAVE}

Educación virtual, pensamiento crítico, foro de discusión

\section{ABSTRACT}

The goal was to determine the influence generated by virtual education on the critical thinking of students during the pandemic. The design incorporated positivist paradigm, applied type, explanatory level, quantitative approach, hypothetical-deductive method and quasi-experimental design elements. The sample was non-probabilistic and consisted of 68 students, divided into an experimental group with 35 students, and a control group with 33 students. Both groups were given a pre-test composed of 20 items with argumentative texts, in order to evaluate their critical thinking. The control group was administered traditional teaching in virtual mode, and the experimental group the virtual teaching program through the discussion forum. A similar test was applied to both groups in the post-test phase. Following the application of the program, the results obtained by means of the Mann Whitney $U$ test (81.00, Z=-6.142<.05) were significant. Conclusion: Virtual education significantly influences critical thinking.

\section{KEYWORDS}

Virtual education; critical thinking; discussion forum

\section{Introducción}

En los últimos años, el avance de la tecnología ha dado saltos exponenciales en los diversos contextos del quehacer humano. La educación, uno de los pilares fundamentales en la formación profesional y como persona, no ha sido ajena a estos avances tecnológicos. Por ello, en el actual tiempo de pandemia, la educación virtual permite a los tutores virtuales llevar una forma de enseñanza a distancia y fomentar así otra manera de educación, donde el discente es el eje principal de su aprendizaje, de su formación personal y profesional, a fin de promover el desarrollo de su pensamiento (Stromquist y Monkman, 2014). Estas formas de desarrollo cognitivo involucran cierta jerarquía de discernimiento, y modifican al estudiante de educación superior en un ser activo, opinante y juicioso, estimulado por el acelerado progreso digitalizado y científico (Halmin y Mokhtar, 2015). 
Al respecto, la Unesco (2013) señaló que se perciben tiempos de cambios tecnológicos que transforman de modo acelerado las interrelaciones de las sociedades. Se trata de una consecuencia de las nuevas formas de conectividad que producen modificaciones económicas, políticas, culturales y educativas, denominadas globalización. La tecnología digital se hace presente en todas las actividades y colabora con los cambios generados en el trabajo, la familia y la educación, buscando siempre el bienestar para los estudiantes. Por ende, los estudiantes utilizan la tecnología digital para el recojo de información y transformarla en conocimiento. Este conocimiento se construye sobre la base del pensamiento crítico de los estudiantes. En este sentido, la Organización para la Cooperación y el Desarrollo Económico (OCDE, 2016) dio a conocer los resultados obtenidos a través de la prueba PISA (2015), que valoró las competencias en comprensión lectora y mostró que el desempeño académico de los alumnos peruanos de 15 años del nivel secundario es preocupante.

Sobre lo mencionado líneas arriba, en el contexto nacional se evidenció que los resultados en comprensión lectora de los estudiantes de 15 años de los últimos años del nivel secundario, según la Evaluación Censal de Estudiantes (ECE, 2018) que realiza cada año el Ministerio de Educación del Perú, se ubicaron en un nivel de inicio, lo cual refleja un aprendizaje no satisfactorio de acuerdo con las expectativas planteadas. Estas debilidades muy notorias en comprensión de lectura se dieron en textos narrativos, expositivos.

En el contexto local, los estudiantes universitarios de diversas carreras del segundo ciclo de una universidad privada de Lima, en la cual se desarrolló el estudio de investigación, no son ajenos a esta problemática. En los exámenes de Comunicación II, tomados con preguntas abiertas de opinión en textos argumentativos, demostraron poca capacidad argumentativa en los niveles de análisis, inferencia, argumentación y solución de problemas del pensamiento crítico. Por tal motivo, se propone emplear estrategias metodológicas educativas utilizando herramientas digitales, aplicadas en un programa de educación virtual con temas controversiales en textos argumentativos. Estas estrategias fomentarán el desarrollo de la argumentación crítica en los estudiantes, para evitar que las secuelas de comprensión de lectura traídas del nivel secundario continúen en educación superior universitaria.

En cuanto a investigaciones internacionales y nacionales se puede aludir la de Ahumada y De la Hoz (2019), quienes mencionaron que la educación virtual se desarrolla de manera semipresencial y virtual desde hace mucho tiempo en el nivel superior y en diferentes partes del mundo. La investigación fue aplicada en el contexto colombiano, con la finalidad de 
determinar el efecto de las herramientas MOOC en el proceso del pensamiento opinante de los discentes. Trabajo cuantitativo, de diseño cuasiexperimental, añade que la implementación de los $\mathrm{MOOC}$ mejora el nivel de desarrollo en las distintas dimensiones de pensamiento crítico. Además, el estudio patentizó la importancia de llevar a cabo la implementación de programas educativos virtuales.

En la realidad chilena, Tabares, Betancourth y Martínez (2019) presentaron un trabajo cuasiexperimental, cuyo propósito fue analizar los efectos de un programa de intervención, basado en el debate sobre el pensamiento opinante de estudiantes de educación superior universitaria. Después de la aplicación de los instrumentos se demostró una mejora significativa en el pensamiento opinante de los discentes, y se concluyó que el debate crítico es una estrategia que privilegia el progreso del juicio opinante en universitarios. Finalmente, el estudio permite aseverar que existen programas de implementación educativa que han evidenciado efectos positivos a favor del educando, como es el caso exitoso y replicable de la realidad chilena.

En Portugal, Alves, Miranda y Morais (2017) desarrollaron un estudio de investigación que tuvo como finalidad obtener indicadores para entender las relaciones entre las plataformas digitales y el rendimiento académico de los discentes universitarios de una institución pública. Concluyeron que hay indicadores parcialmente positivos en cuanto al ingreso de los estudiantes a un entorno virtual: a mayor número de accesos a la plataforma, mayor número de estudiantes aprobados. Se puede afirmar de manera relativa que, en cuanto a la cantidad de accesos a las plataformas virtuales, el estudiante reflejará estos ingresos en la aprobación de sus cursos; sin embargo, la cantidad de accesos no garantiza un rendimiento académico categórico.

En el contexto de Ecuador, Prada (2016) realizó una investigación de enfoque mixto y tuvo como objetivo determinar la incidencia de la plataforma «Más tecnología» sobre el pensamiento. Realizó una medición cuantitativa por medio de un pretest y un postest a través de un inventario de competencias y la evaluación cualitativa aplicando una plataforma virtual. Estos instrumentos midieron las dimensiones del pensamiento crítico propuesto por Facione, análisis, inferencia, argumentación y solución de problemas. Concluyó que la plataforma virtual promueve habilidades de flexibilidad, competencias para formular juicios basados en comunicación concreta y en la clasificación, categorización y reflexión de la información. Así, se corrobora que la implementación de una plataforma virtual en instituciones educativas debe ser mediada por el docente en clases sincrónicas con los estudiantes; luego, ellos utilizan la plataforma para el 
desarrollo de actividades prácticas, con materiales didácticos propuestos en esta, que fortalecen las competencias y capacidades del pensamiento crítico de los estudiantes.

En relación con los trabajos nacionales, Álvarez (2020) realizó un estudio de carácter cuasiexperimental, con el objetivo de determinar el efecto del debate académico en el pensamiento opinante en los discentes. Demostró, por medio de resultados estadísticos, que el debate académico creó un efecto significativo en el pensamiento crítico de los estudiantes. Esta experiencia investigativa genera expectativas para ser aplicada en la educación virtual. En la misma línea está el trabajo investigativo de López (2019), quien desarrolló una tesis cuantitativa, descriptiva correlacional. El objetivo fue instaurar la correlación entre el aula virtual Blackboard y el pensamiento crítico en el curso de Análisis de Circuitos Eléctricos. López concluyó que el uso de esta plataforma mejoró las habilidades críticas de los estudiantes del nivel superior concernientes a la argumentación y las propuestas de solución de problemas del entorno. En el mismo sentido, Rojas (2019) desarrolló una tesis que consistió en la elaboración de un programa contextualizado tomando la metodología, con el objetivo de mejorar la capacidad argumentativa, la relevancia de la fuente de información, las propuestas y las alternativas viables en estudiantes del nivel superior. Se utilizó el diseño crítico propositivo y se concluyó que la metodología Boisvert mejoró significativamente las deficiencias del pensamiento crítico. Por tanto, el autor recomienda la elaboración de un programa que mejore la criticidad en los estudiantes.

Por otro lado, Huapaya (2016) desarrolló una tesis de enfoque cuantitativo, aplicada, de tipo experimental, cuyo propósito fue establecer si la educación virtual, como una metodología de modalidad a distancia en la enseñanza-aprendizaje enfocada de manera didáctica, influye positivamente en el proceso formativo en los futuros ingenieros de las instituciones superiores universitarias. Su conclusión sostiene que es importante crear un paradigma educativo digital con una estructura que agrupe elementos educativos imprescindibles para una educación virtual. De este modo, permitirán que las TIC contribuyan de manera positiva en un aprendizaje autónomo en su formación profesional.

Asimismo, es preponderante mencionar el trabajo de Cuadrao (2016), quien desarrolló su investigación bajo el diseño experimental, tipo cuasiexperimental, cuyo objetivo fue establecer el impacto de la aplicación del método de enseñanza virtual Blended Learning. Llegó a la conclusión de que el aprendizaje en las dimensiones de su investigación con este método presentó un aumento significativo. 
A su vez, las teorías relacionadas con la variable pensamiento crítico se cimentan en la teoría sociocultural y cognitiva y la base teórica de Facione. Al respecto, Facione (2007) menciona que los seres humanos recurren al pensamiento crítico para resolver problemas o tomar decisiones, ya que, a través de él, se dan discernimientos juiciosos de lo que se cree o se construye. En relación con el enfoque sociocultural, Vygotsky (1997) señaló que entre el pensamiento y el lenguaje, el proceso mental y las palabras existe una relación muy estrecha; entonces, el fundamento es la interrelación que tiene el ser humano con sus semejantes, es decir, el proceso cognitivo personal se origina de la sociedad. Este es el sustento de esta teoría de aprendizaje. Las interacciones sociales entre los estudiantes en las realizaciones de quehaceres académicos y el docente, cuando este gestiona, monitorea y los dirige en el desarrollo de sus competencias, las realiza por medio de un conjunto de actividades sociales externas. Estas acciones pedagógicas son facilitadas por este enfoque teórico. La interacción entre la realidad social y la comunicación es fundamental para el desarrollo del pensamiento crítico. Este no solamente será el lugar de aprendizaje, sino también estímulo de creación y elaboración de propuestas de soluciones creativas para los distintos inconvenientes que se presenten en la realidad.

Con respecto al enfoque cognitivo, Fierro (2011) expuso que los procesos mentales guiados al procesamiento de la información (en los que están comprometidas la percepción, la memoria, la planificación, la toma de decisiones y la generación de un nuevo aprendizaje) son el propósito fundamental de estudio de esta teoría.

Por su parte, Facione (2007) definió al pensamiento crítico como una secuencia de realización de discernimientos connotados y juiciosos sobre lo que se cree o lo que se construye, y es concretado por los seres para resolver problemas y tomar decisiones. Asimismo, divide al pensamiento crítico en cuatro magnitudes: análisis, inferencia, argumentación y formulación de propuestas de solución. En la investigación estas magnitudes fueron utilizadas como dimensiones de la variable dependiente.

En el estadio de la dimensión de análisis, la información de las lecturas es procesada en un primer nivel denominado explícito, donde las ideas, los datos y los nombres se encuentran de manera expresa. En esta dimensión se ubican las habilidades de identificar y reconocer el tema, las ideas principales, secundarias o terciarias. Además, se pueden reconocer las partes o los componentes de un texto argumentativo y descubrir los elementos que posee cada uno, como los subtemas.

En la dimensión de inferencia se procesa la información de manera implícita, encauzando diversas habilidades. En primer lugar, se presenta 
la habilidad de inferir, colegir, deducir, interpretar, que se obtienen de los datos no explícitos de los textos. Con ellos también se puede comparar o establecer semejanzas y diferencias entre dos ideas o más; resumir y sintetizar las ideas principales de un texto; e interpretar y parafrasear con un lenguaje sencillo y comprensible. Por último, para adquirir esta habilidad se necesita haber desarrollado la fase de análisis.

La tercera dimensión es el estadio de argumentación, la fase donde se formulan posturas a favor o en contra de un tema controversial. Se trata quizá del nivel más alto del pensamiento crítico, donde el estudiante es capaz de analizar, debatir y evaluar, y emplea todas las habilidades adquiridas en la fase inferencial. En esta dimensión se encuentra la habilidad de argumentar, es decir, defender los puntos de vista a favor o en contra, con base en estrategias argumentativas de autoridad, datos, hechos.

La cuarta dimensión es el estadio de formulación de propuestas de solución. Es la fase donde el estudiante debe ser capaz de buscar propuestas de solución ante cualquier problema que se le presente o se le formule.

Por su parte, las teorías relacionadas con la variable independiente educación virtual se sustentan en la definición teórica de Gros (2011), quien la define como una metodología educativa a distancia que utiliza herramientas tecnológicas emergentes. Está integrada por recursos de aprendizaje, soporte técnico virtual y acompañamiento del docente. Asimismo, esta variable se vincula con la teoría del constructivismo, que centra al estudiante en un ser protagónico, activo, dinámico; es decir, es el eje central del aprendizaje. En consecuencia, el enfoque más utilizado de estas teorías en el diseño de ambientes instruccionales es el constructivista, ya que el estudiante construye sus aprendizajes. En la última década ha surgido la tecnología, que ha influido en todas las actividades del ser humano. En este contexto, surge una nueva teoría, el conectivismo, para explicar el aprendizaje de la mano de las herramientas digitales y la comunicación. En cuanto al conectivismo, Siemens (2004) sustenta que esta teoría de aprendizaje se basa en los principios de la teoría del caos, que relaciona la complejidad de las redes neuronales y la autoorganización de conexiones en una red. Estas conexiones pueden ser personas, conceptos, ideas, cosas diferentes. Un aspecto fundamental del conectivismo es la comparación de una malla con nodos y conexiones. Es una nueva teoría de aprendizaje para la era digital.

En la actualidad, con el uso de las nuevas estrategias didácticas se deja de lado el protagonismo del docente y se le centra en el estudiante. Sobre lo expuesto, Santos (2015) señala que en la educación actual se asumen nuevos roles en la modalidad tecnológica virtual. Así, se da a conocer la importancia que está asumiendo el estudiante, quien dejará de ser un 
espectador para constituirse en principal actor de su aprendizaje. En consecuencia, el docente, que era el actor principal en la educación tradicional, pasará a un segundo plano.

En el mundo del conocimiento, la tecnología ocupa un lugar especial en todos los ámbitos de la vida del ser humano. Solo se debe orientar a propósitos positivos relacionados con la formación educativa y cultural del hombre para el bien de la sociedad. En consideración a lo expresado, Gros (2011), como Ardoin, Clark y Kelsey (2013), mencionan que la educación virtual se considera como una actividad formativa y su diseño debe estar direccionado según las necesidades de los discentes, con el fin de obtener las habilidades y capacidades previstas. Al respecto, Gros considera que todo modelo debe constar de tres componentes sustanciales: (a) los materiales de aprendizaje, (b) la cooperación y (c) el acompañamiento. Estos tres elementos influyen de manera significativa en la actividad de aprendizaje. Ello proporcionará las orientaciones digitales que se ejecutarán de modo más óptimo y eficiente en los alumnos. Es de suma importancia el acompañamiento desde el inicio del módulo, así como realizar un recordatorio en mitad de semana y dar el mensaje de cierre. Este acompañamiento virtual se realiza durante todo el avance del ciclo.

En el objetivo de la investigación se formuló determinar la influencia que genera la educación virtual en el pensamiento crítico. La hipótesis general es que la educación virtual influye significativamente en el pensamiento crítico en los estudiantes de una universidad privada de Lima.

\section{Materiales y método}

El presente trabajo de investigación es de tipo aplicado y se desarrolló en el paradigma positivista. Según Samaja (2012), toda forma de conocimiento vivencial se cimenta en la experiencia sensorial y la observación. El experimento tiende a buscar el origen de los fenómenos sociales, y ello se asocia con el método científico. Se utilizó el diseño cuasiexperimental. Según Campbelt y Stanley (1995), los diseños cuasiexperimentales generalmente se aplican en la investigación educativa y están conformados por dos grupos, uno llamado experimental y otro, control. El diseño de la investigación fue:

$$
\begin{array}{llll}
\text { GE: } & 0_{1} & X & 0_{2} \\
\text { GC: } & 0_{3} & -- & 0_{4}
\end{array}
$$

Donde:

$$
\begin{aligned}
& \text { GE: grupo experimental } \\
& \text { GC: grupo control } \\
& \text { X: aplicación de la educación virtual }
\end{aligned}
$$




\section{$0_{n}: n=1 ; 3$ : observaciones antes \\ $\mathrm{n}=2 ; 4$ : observaciones después}

La población estuvo conformada por 2150 estudiantes del segundo ciclo (2020-1) en una universidad privada de Lima. López (2013) refirió que la población es el conjunto total de individuos visibles en un área determinada.

TABLA 1. Población de estudio

\begin{tabular}{lc} 
& Facultades \\
Comunicaciones & 770 \\
\hline Administración y Negocios Internacionales & 510 \\
\hline Arquitectura y Urbanismo & 620 \\
\hline Contabilidad & 250 \\
\hline Total & 2150 \\
\hline
\end{tabular}

Fuente: matriculados en el periodo académico 2020

Se trató de una muestra conocida y comprendida por 68 estudiantes matriculados en el primer ciclo 2020-1 en el curso de Comunicación II de las facultades de Arquitectura y Urbanismo, Ciencias de la Comunicación y Administración, en una universidad privada de Lima, que correspondieron al grupo experimental $(\mathrm{GE}=35)$ y al grupo control $(\mathrm{GC}=33)$. Valderrama (2015) sostiene que la muestra no probabilística intencional es aquella que ya está establecida con anterioridad, y por razones de criterios del investigador la emplea para el estudio.

El muestreo fue del tipo no probabilístico, debido a que la muestra, desde un inicio, estuvo asignada para el dictado de clases y por conveniencia se designó para el GE el aula A y para el GC el aula B, de manera intencional o a criterio del investigador. Carrasco (2017) indicó que en las muestras intencionadas el investigador elige a su muestra según su criterio, sin tener en consideración ninguna regla estadística.

TABLA 2. Muestra de estudio

\begin{tabular}{lll} 
& Secciones & Estudiantes \\
Grupo experimental (GE) & Aula A & 35 \\
\hline Grupo control (GC) & Aula B & 33 \\
\hline Total & & 68 \\
\hline
\end{tabular}

Fuente: matriculados en el periodo académico 2020 


\section{Técnicas e instrumentos de recolección de datos, validez y confiabilidad}

La técnica utilizada fue la observación de análisis documentario, ya que se visualizaron las respuestas en el foro de discusión de la plataforma Blackboard. Para la recolección y evaluación de los datos se elaboró la rúbrica para el pretest y el postest. La rúbrica evaluó 20 preguntas a fin de medir la variable pensamiento crítico. Esta técnica se realiza, en forma organizada y precisa, para construir las relaciones entre las variables, los objetivos y los procedimientos (Quezada, 2015). El objetivo del instrumento fue medir el nivel de pensamiento crítico. La administración fue individual y utilizó la herramienta virtual del foro de discusión, en un tiempo de 60 minutos aproximadamente, durante la semana programada. La prueba está constituida por 20 ítems, divididos en cuatro dimensiones con sus indicadores, con respuestas subjetivas y un puntaje vigesimal de 0 a 20.

Para probar la validez del instrumento de pensamiento crítico, se utilizó la prueba de $V$ de Aiken por medio de la validez de cinco jueces. A cada uno se le hizo llegar un formato de validación de instrumento. Después de analizar dieron su veredicto de pertinencia, relevancia y claridad. Sánchez y Reyes (2015) indicaron que el instrumento debe medir lo que se desea medir, que pueda evidenciar efectivamente la capacidad de medición.

Con respecto a la confiabilidad, Hernández y Mendoza (2018) indicaron que la fiabilidad del instrumento es una manera de verificación para saber la consistencia y la coherencia de los ítems del instrumento. Antes de iniciar la investigación se ejecutó un sondeo piloto con 30 estudiantes. Luego de este sondeo, no se realizó ninguna modificación a la prueba piloto, por lo que se compiló la información referente a la variable. Posteriormente, se anotaron los datos en una hoja de cálculo de Excel y el programa SPSS versión 25; con la prueba alfa de Cronbach se verificó la confiabilidad del instrumento y se obtuvo como resultado un ,866. Se usó esta técnica porque el instrumento de pensamiento crítico presenta preguntas politómicas. La prueba alfa de Cronbach comprobó que los instrumentos presentan muy alta confiabilidad. Por tal razón, se utilizaron en el pretest $y$ el postest $y$ son fiables.

TABLA 3. Estadística de fiabilidad del pensamiento crítico

\begin{tabular}{cc} 
Alfa de Cronbach & Nro. de elementos \\
\hline, 866 & 20 \\
\hline
\end{tabular}

Fuente: elaboración propia 
La tabla 3 expone el valor de confiabilidad del instrumento (de ,866), cuya interpretación es excelente.

\section{Procedimiento}

Se recogió la información de la variable de estudio a través de dos evaluaciones aplicadas a los estudiantes, según la venia informada previa a la aplicación. Se recogió en dos periodos. El primer momento fue cuando se aplicó el pretest para el GC y el GE, y se implementó la propuesta de educación virtual en el grupo experimental en 12 sesiones. A continuación, en el segundo momento, se aplicó el postest también a ambos grupos. Finalmente, se tabularon los datos en una hoja de Excel y luego se pasaron al programa estadístico SPSS versión 25.

\section{Métodos de análisis de datos}

Para la estadística descriptiva se consiguieron cantidades y porcentajes de los datos obtenidos de forma general del pensamiento crítico y sus dimensiones, a través del programa estadístico SPSS 25. Se mostró la información del grupo control y del grupo experimental. En relación con el análisis descriptivo, Vargas (2014) manifestó que esta etapa es indispensable, debido a que da sentido a lo recogido en el proceso, al tratarlo y organizarlo de manera adecuada para explicar e interpretar el objeto de la investigación y responder al planteamiento inicial.

En cuanto al análisis inferencial del estudio, para la comparación de hipótesis y en el caso donde la variable no presenta distribución normal, esta se predispone a la utilización de la prueba no paramétrica. La prueba de Shapiro-Wilk se empleó para indagar si los datos procedían de una distribución normal. Para el contraste de las hipótesis, se empleó la prueba $U$ de Mann-Whitney para grupos independientes.

\section{Resultados}

\section{Resultados descriptivos}

\section{Descripción del pensamiento crítico}

En la tabla 4 y la figura 1 se exponen los niveles del pensamiento crítico del GE y GC tanto del pretest y postest. Se observó en el pretest GC tiene un porcentaje del 33,3\% para el nivel en inicio, 63,6\% para en proceso y 3,1\% para el nivel logrado, mientras que para el GE tiene el $22,9 \%$ en el nivel en inicio, $74,3 \%$ en el nivel en proceso y $2,8 \%$ en logrado en sus calificaciones. Además, para el postest del GC Ilegó al 3,0\% en inicio, 78,8\% en proceso y 18,2\% en el nivel logrado, mientras que para el GE llegó al $11,4 \%$ en proceso, $82,93 \%$ en el nivel logrado y $5,7 \%$ en el nivel sobresaliente. Ello indica el logro de la competencia del pensamiento crítico en 
los niveles, resultados obtenidos después de la aplicación del programa de educación virtual, empleando la plataforma Blackboard.

TABLA 4. Niveles del pensamiento crítico pretest y postest

\begin{tabular}{|c|c|c|c|c|c|c|c|}
\hline & & & En inicio & En proceso & Logrado & Sobresaliente & Total \\
\hline \multirow{4}{*}{ 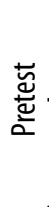 } & \multirow{2}{*}{ Grupo control } & Frecuencia & 11 & 21 & 1 & 0 & 33 \\
\hline & & Porcentaje & 33,3 & 63,6 & 3,1 & 0 & 100 \\
\hline & \multirow{2}{*}{$\begin{array}{l}\text { Grupo } \\
\text { experimental }\end{array}$} & Frecuencia & 8 & 26 & 1 & 0 & 35 \\
\hline & & Porcentaje & 22,9 & 74,3 & 2,8 & 0 & 100 \\
\hline \multirow{4}{*}{ 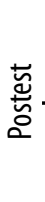 } & \multirow{2}{*}{ Grupo control } & Frecuencia & 1 & 26 & 6 & 0 & 33 \\
\hline & & Porcentaje & 3,0 & 78,8 & 18,2 & 0 & 100 \\
\hline & \multirow{2}{*}{$\begin{array}{l}\text { Grupo } \\
\text { experimental }\end{array}$} & Frecuencia & 0 & 4 & 29 & 2 & 35 \\
\hline & & Porcentaje & 0 & 11,4 & 82,9 & 5,7 & 100 \\
\hline
\end{tabular}

Fuente: elaboración propia

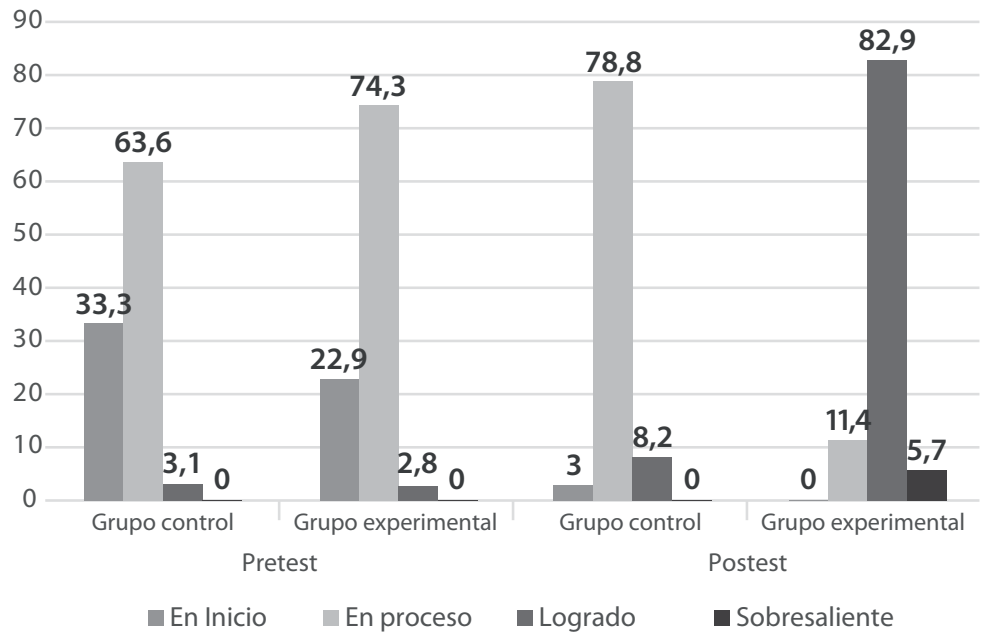

FIGURA 1. Niveles de pensamiento crítico pretest y postest

\section{Descripción de la dimensión análisis del pensamiento crítico}

La tabla 5 expone los niveles de la dimensión análisis del pensamiento crítico del GE y GC del pretest y el postest. Se observó en el pretest que el GC tiene un repunte del $48,4 \%$ del nivel logrado, mientras que para el GE tiene un nivel del $57,2 \%$ en el nivel sobresaliente en sus calificaciones. Además, el postest del GC asciende a un nivel logrado del $57,6 \%$ en relación con el GE, que obtuvo 48,6\% para el nivel sobresaliente. Ello indica el logro de la competencia del pensamiento crítico en la dimensión de análisis, resultados obtenidos, después de la aplicación del programa educación virtual empleando la plataforma Blackboard. 


\section{Descripción de la dimensión inferencia del pensamiento crítico}

La tabla 6 expone los niveles de la dimensión inferencia del pensamiento crítico del GE y GC del pretest y el postest. Se observó en el pretest que el GC tiene un porcentaje del $57,6 \%$ para el nivel en inicio, mientras que el GE tiene el $80,0 \%$ para el nivel en inicio en sus calificaciones. Además, el postest del GC se mantiene casi con las mismas proporciones en relación con el GE, que llegó hasta el 31,4\% en logrado y el $45,7 \%$ para el nivel sobresaliente. Esto indica el logro de la competencia del pensamiento crítico en la dimensión de inferencia, resultados obtenidos después de la aplicación del programa de educación virtual, empleando la plataforma Blackboard.

TABLA 5. Niveles de la dimensión análisis pretest y postest

\begin{tabular}{|c|c|c|c|c|c|c|c|}
\hline & & & En inicio & En proceso & Logrado & Sobresaliente & Total \\
\hline \multirow{4}{*}{ 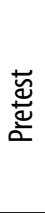 } & \multirow{2}{*}{$\begin{array}{l}\text { Grupo } \\
\text { control }\end{array}$} & Frecuencia & 2 & 13 & 6 & 2 & 33 \\
\hline & & Porcentaje & 6,1 & 39,4 & 48,4 & 6,1 & 100 \\
\hline & \multirow{2}{*}{$\begin{array}{l}\text { Grupo } \\
\text { experimental }\end{array}$} & Frecuencia & 2 & 6 & 7 & 20 & 35 \\
\hline & & Porcentaje & 5,7 & 17,1 & 20,0 & 57,2 & 100 \\
\hline \multirow{4}{*}{ 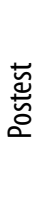 } & \multirow{2}{*}{$\begin{array}{l}\text { Grupo } \\
\text { control }\end{array}$} & Frecuencia & 1 & 10 & 19 & 3 & 33 \\
\hline & & Porcentaje & 3,0 & 30,3 & 57,6 & 9,1 & 100 \\
\hline & \multirow{2}{*}{$\begin{array}{l}\text { Grupo } \\
\text { experimental }\end{array}$} & Frecuencia & 1 & 2 & 15 & 17 & 35 \\
\hline & & Porcentaje & 2,9 & 5,6 & 42,9 & 48,6 & 100 \\
\hline
\end{tabular}

Fuente: elaboración propia

TABLA 6. Niveles de la dimensión inferencia pretest y postest

\begin{tabular}{|c|c|c|c|c|c|c|c|}
\hline & & & En inicio & En proceso & Logrado & Sobresaliente & Total \\
\hline \multirow{4}{*}{ } & \multirow{2}{*}{$\begin{array}{l}\text { Grupo } \\
\text { control }\end{array}$} & Frecuencia & 19 & 10 & 4 & & 33 \\
\hline & & Porcentaje & 57,6 & 30,3 & 12,1 & 0 & 100 \\
\hline & \multirow{2}{*}{$\begin{array}{l}\text { Grupo } \\
\text { experimental }\end{array}$} & Frecuencia & 28 & 4 & 3 & & 35 \\
\hline & & Porcentaje & 80,0 & 11,4 & 8,6 & 0 & 100 \\
\hline \multirow{4}{*}{ 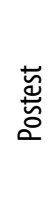 } & \multirow{2}{*}{$\begin{array}{l}\text { Grupo } \\
\text { control }\end{array}$} & Frecuencia & 17 & 11 & 4 & 1 & 33 \\
\hline & & Porcentaje & 51,5 & 33,3 & 12,1 & 3,1 & 100 \\
\hline & \multirow{2}{*}{$\begin{array}{l}\text { Grupo } \\
\text { experimental }\end{array}$} & Frecuencia & 1 & 7 & 11 & 16 & 35 \\
\hline & & Porcentaje & 2,9 & 20,0 & 31,4 & 45,7 & 100 \\
\hline
\end{tabular}

Fuente: elaboración propia 


\section{Descripción de la dimensión argumentación del pensamiento crítico}

La tabla 7 expone los niveles de la dimensión argumentación del pensamiento crítico del GE y GC del pretest y el postest. Se observó en el pretest que el GC tiene un porcentaje del $45,5 \%$ para el nivel en logrado y $3,0 \%$ para sobresaliente, mientras que el GE tiene el $42,9 \%$ para el nivel logrado y $2,9 \%$ para el sobresaliente en sus calificaciones. Además, el postest del GC Ilegó al $48,5 \%$ en el nivel logrado y al $12,1 \%$ en el sobresaliente, mientras que para el GE llegó al 45,7\% en logrado y al 37,1\% en el nivel sobresaliente. Esto indica el logro de la competencia del pensamiento crítico en la dimensión de argumentación, resultados obtenidos después de la aplicación del programa educación virtual, empleando la plataforma Blackboard.

TABLA 7. Niveles de la dimensión argumentación pretest y postest

\begin{tabular}{|c|c|c|c|c|c|c|c|}
\hline & & & En inicio & En proceso & Logrado & Sobresaliente & Total \\
\hline \multirow{4}{*}{ 芯 } & \multirow{2}{*}{$\begin{array}{l}\text { Grupo } \\
\text { control }\end{array}$} & Frecuencia & 11 & 6 & 15 & 1 & 33 \\
\hline & & Porcentaje & 33,3 & 18,2 & 45,5 & 3,0 & 100 \\
\hline & \multirow{2}{*}{$\begin{array}{l}\text { Grupo } \\
\text { experimental }\end{array}$} & Frecuencia & 6 & 13 & 15 & 1 & 35 \\
\hline & & Porcentaje & 17,1 & 37,1 & 42,9 & 2,9 & 100 \\
\hline \multirow{4}{*}{$\begin{array}{l}\overleftarrow{\leftrightarrows} \\
\overleftarrow{\Delta}\end{array}$} & \multirow{2}{*}{$\begin{array}{l}\text { Grupo } \\
\text { control }\end{array}$} & Frecuencia & 6 & 7 & 16 & 4 & 33 \\
\hline & & Porcentaje & 18,2 & 21,2 & 48,5 & 12,1 & 100 \\
\hline & \multirow{2}{*}{$\begin{array}{l}\text { Grupo } \\
\text { experimental }\end{array}$} & Frecuencia & 1 & 5 & 16 & 13 & 35 \\
\hline & & Porcentaje & 2,9 & 14,3 & 45,7 & 37,1 & 100 \\
\hline
\end{tabular}

Fuente: elaboración propia

TABLA 8. Niveles de la dimensión formulación de propuestas de solución pretest y postest

\begin{tabular}{|c|c|c|c|c|c|c|c|}
\hline & & & En inicio & En proceso & Logrado & Sobresaliente & Total \\
\hline \multirow{4}{*}{ 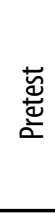 } & \multirow{2}{*}{$\begin{array}{l}\text { Grupo } \\
\text { control }\end{array}$} & Frecuencia & 17 & 11 & 5 & & 33 \\
\hline & & Porcentaje & 51,5 & 33,3 & 15,2 & 0 & 100 \\
\hline & \multirow{2}{*}{$\begin{array}{l}\text { Grupo } \\
\text { experimental }\end{array}$} & Frecuencia & 26 & 7 & 2 & & 35 \\
\hline & & Porcentaje & 74,3 & 20,0 & 5,7 & 0 & 100 \\
\hline \multirow{4}{*}{ 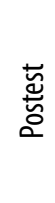 } & \multirow{2}{*}{$\begin{array}{l}\text { Grupo } \\
\text { control }\end{array}$} & Frecuencia & 7 & 11 & 8 & 7 & 33 \\
\hline & & Porcentaje & 21,2 & 33,3 & 24,3 & 21,2 & 100 \\
\hline & \multirow{2}{*}{$\begin{array}{l}\text { Grupo } \\
\text { experimental }\end{array}$} & Frecuencia & 3 & 8 & 18 & 6 & 35 \\
\hline & & Porcentaje & 8,6 & 22,9 & 51,4 & 17,1 & 100 \\
\hline
\end{tabular}

Fuente: elaboración propia 


\section{Descripción de la dimensión formulación de propuestas de solución del pensamiento crítico}

La tabla 8 expone los niveles de la dimensión formulación de propuestas de solución del pensamiento crítico del GE y GC del pretest y el postest. Se observó en el pretest que el GC tiene un porcentaje del 51,5\% para el nivel en inicio, mientras que el GE tiene el $74,3 \%$ para nivel en inicio en sus calificaciones. Además, el postest del GC llega al $24,3 \%$ en el nivel logrado y al $21,2 \%$ en el sobresaliente, mientras que para el GE llegó al $51,4 \%$ en logrado y al $17,1 \%$ en el nivel sobresaliente. Esto indica el logro de la competencia del pensamiento crítico en la dimensión formulación de propuestas de solución, resultados obtenidos después de la aplicación del programa educación virtual, empleando la plataforma Blackboard.

\section{Resultados inferenciales}

\section{Prueba de normalidad}

Se precisó la prueba de normalidad de las calificaciones obtenidas por el grupo experimental y grupo control pretest y postest, respectivamente.

La tabla 9 exhibe los valores de la prueba de bondad de ajuste de Kolmogórov-Smirnov, para los puntajes obtenidos en el GC y GE antes y después, respectivamente. Los datos obtenidos del $p$-valor $p<, 05$ indican la no aproximación a una distribución normal; en consecuencia, se aplicaron estadísticos no paramétricos. Específicamente, la prueba U de MannWhitney para grupos independientes y la de rangos de Wilcoxon para grupos relacionados. Al respecto, Pérez y López (2008) detallaron que si el valor del $p$-valor es menor a nivel de significancia de 0,05 , entonces los datos registrados tienen distribución no normal.

TABLA 9. Prueba de normalidad del GC y GE pretest y postet

\begin{tabular}{lccc} 
& \multicolumn{3}{c}{ Kolmogórov-Sminov } \\
GEPRE &, 183 & Gl & Sig. \\
\hline GEPOS &, 204 & 35 &, 004 \\
\hline GCPRE &, 145 & 35 &, 001 \\
\hline GCPOS &, 197 & 33 &, 075 \\
\hline
\end{tabular}

Fuente: elaboración propia 


\section{Contraste de la hipótesis general}

\section{Prueba de hipótesis general}

$\mathrm{H}_{0}$ : la educación virtual no influye significativamente en el pensamiento crítico en los estudiantes de una universidad privada de Lima, 2020.

$\mathrm{H}_{\mathrm{a}}$ : la educación virtual influye significativamente en el pensamiento crítico en los estudiantes de una universidad privada de Lima, 2020.

La tabla 10 muestra los valores de contraste para el GE y GC después de la aplicación del programa EV en el pensamiento crítico, empleando la plataforma Blackboard. La prueba de U de Mann-Whitney: (81,00, Z = $-6,142<-1,96)$, con ( $p$-valor $=, 000<, 05$ ). Ante la suficiente evidencia estadística, se afirmó que la educación virtual influye significativamente en el pensamiento crítico en los estudiantes de una universidad privada de Lima, 2020.

\section{Prueba de hipótesis específica 1}

$\mathrm{H}_{0}$ : la educación virtual no influye significativamente en el análisis del pensamiento crítico en los estudiantes de una universidad privada de Lima, 2020.

$\mathrm{H}_{\mathrm{a}}$ : la educación virtual influye significativamente en el análisis del pensamiento crítico en los estudiantes de una universidad privada de Lima, 2020

La tabla 11 muestra los valores de contraste para el GE y GC después de la aplicación en la dimensión análisis del pensamiento crítico empleando la plataforma Blackboard. La prueba de U de Mann-Whitney: (299,50, Z $=-3,713<-1,96)$, con ( $p$-valor $=, 000<, 05$ ). Ante la suficiente evidencia estadística, se afirmó que la educación virtual influye significativamente en el análisis del pensamiento crítico en los estudiantes de una universidad privada de Lima, 2020.

\section{Prueba de hipótesis específica 2}

$\mathrm{H}_{0}$ : la educación virtual no influye significativamente en la inferencia del pensamiento crítico en los estudiantes de una universidad privada de Lima, 2020.

$\mathrm{H}_{\mathrm{a}}$ : la educación virtual influye significativamente en la inferencia del pensamiento crítico en los estudiantes de una universidad privada de Lima, 2020

La tabla 12 muestra los valores de contraste para el GE y GC después de la aplicación en la dimensión inferencia del pensamiento crítico empleando la plataforma Blackboard. La prueba de U de Mann-Whitney: $(136,50, Z=-5,558<-1,96)$, con $(p-$ valor $=, 000<, 05)$. Ante la suficiente 
evidencia estadística, se afirmó que la educación virtual influye significativamente en la inferencia del pensamiento crítico en los estudiantes de una universidad privada de Lima, 2020.

\section{Prueba de hipótesis específica 3}

$\mathrm{H}_{0}$ : la educación virtual no influye significativamente en la argumentación del pensamiento crítico en los estudiantes de una universidad privada de Lima, 2020.

$\mathrm{H}_{\mathrm{a}}$ : La educación virtual influye significativamente en la argumentación del pensamiento crítico en los estudiantes de una universidad privada de Lima, 2020.

TABLA 10. Prueba U de Mann-Whitney: GC y GE después de aplicar la prueba del pensamiento crítico empleando la plataforma Blackboard

\begin{tabular}{|c|c|c|c|c|c|}
\hline & Grupos & N & Rango promedio & Suma de rangos & U de Mann-Whitney \\
\hline \multirow{3}{*}{ 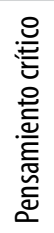 } & $\begin{array}{l}\text { Grupo } \\
\text { experimental }\end{array}$ & 35 & 48,69 & 1704,00 & $\mathrm{U}=81,00$ \\
\hline & $\begin{array}{l}\text { Grupo } \\
\text { control }\end{array}$ & 33 & 19,45 & 642,00 & $Z=-6,142$ \\
\hline & Total & 68 & & & Sig. asíntota (bilateral) $=, 000$ \\
\hline
\end{tabular}

Fuente: elaboración propia

TABLA 11. Prueba U de Mann-Whitney: GC y GE después de aplicar el análisis del pensamiento crítico empleando la plataforma Blackboard

\begin{tabular}{|c|c|c|c|c|c|}
\hline & Grupos & N & Rango promedio & Suma de rangos & U de Mann-Whitney \\
\hline \multirow{3}{*}{ 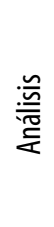 } & $\begin{array}{l}\text { Grupo } \\
\text { experimental }\end{array}$ & 35 & 42,44 & 1485,50 & $U=299,50$ \\
\hline & $\begin{array}{l}\text { Grupo } \\
\text { control }\end{array}$ & 33 & 26,08 & 860,50 & $Z=-3,713$ \\
\hline & Total & 68 & & & Sig. asíntota (bilateral) $=, 000$ \\
\hline
\end{tabular}

Fuente: elaboración propia

TABLA 12. Prueba U de Mann-Whitney: GC y GE después de aplicar la inferencia del pensamiento crítico empleando la plataforma Blackboard

\begin{tabular}{|c|c|c|c|c|c|}
\hline & Grupos & N & Rango promedio & Suma de rangos & U de Mann-Whitney \\
\hline \multirow{3}{*}{ 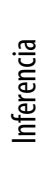 } & $\begin{array}{l}\text { Grupo } \\
\text { experimental }\end{array}$ & 35 & 47,10 & 1648,50 & $U=136,50$ \\
\hline & Grupo control & 33 & 21,14 & 697,50 & $Z=-5,558$ \\
\hline & Total & 68 & & & Sig. asíntota (bilateral) $=, 000$ \\
\hline
\end{tabular}

Fuente: elaboración propia 
TABLA 13. Prueba $U$ de Mann-Whitney: GC y GE después de aplicar la argumentación del pensamiento crítico empleando la plataforma Blackboard.

\begin{tabular}{|c|c|c|c|c|c|}
\hline & Grupos & $\mathbb{N}$ & Rango promedio & Suma de rangos & U de Mann-Whitney \\
\hline \multirow{3}{*}{ 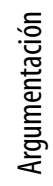 } & $\begin{array}{l}\text { Grupo } \\
\text { experimental }\end{array}$ & 35 & 40,56 & 1419,50 & $U=365,50$ \\
\hline & Grupo control & 33 & 28,08 & 926,50 & $Z=-2,783$ \\
\hline & Total & 68 & & & Sig. asíntota (bilateral) $=, 005$ \\
\hline
\end{tabular}

Fuente: elaboración propia

La tabla 13 muestra los valores de contraste para el GE y GC después de la aplicación en la dimensión argumentación del pensamiento crítico empleando la plataforma Blackboard. La prueba de $U$ de Mann-Whitney: $(365,50, Z=-2,783<-1,96)$, con ( $p$-valor $=, 005<, 05)$. Ante la suficiente evidencia estadística, se afirmó que la educación virtual influye significativamente en la argumentación del pensamiento crítico en los estudiantes de una universidad privada de Lima, 2020.

\section{Prueba de hipótesis específica 4}

$\mathrm{H}_{0}$ : la educación virtual no influye significativamente en la formulación de propuestas de solución del pensamiento crítico en los estudiantes de una universidad privada de Lima, 2020.

$\mathrm{H}_{\mathrm{a}}$ : la educación virtual influye significativamente en la formulación de propuestas de solución del pensamiento crítico en los estudiantes de una universidad privada de Lima, 2020.

La tabla 14 muestra los valores de contraste para el GE y GC después de la aplicación en la dimensión formulación de propuestas de solución del pensamiento crítico empleando la plataforma Blackboard. La prueba de $U$ de Mann-Whitney: $(470,500, Z=-1,373>-1,96)$, con ( $p$-valor $=, 170>, 05)$. Ante la suficiente evidencia estadística, se afirmó que la educación virtual no influye en la formulación de propuestas de solución del pensamiento crítico en los estudiantes de una universidad privada de Lima, 2020.

TABLA 14. Prueba U de Mann-Whitney: GC y GE después de aplicar la formulación de propuestas de solución del pensamiento crítico empleando la plataforma Blackboard

\begin{tabular}{|c|c|c|c|c|c|}
\hline & Grupos & N & $\begin{array}{l}\text { Rango } \\
\text { promedio }\end{array}$ & $\begin{array}{l}\text { Suma de } \\
\text { rangos }\end{array}$ & U de Mann-Whitney \\
\hline \multirow{3}{*}{ 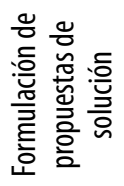 } & $\begin{array}{l}\text { Grupo } \\
\text { experimental }\end{array}$ & 35 & 37,56 & 1314,50 & $U=470,50$ \\
\hline & Grupo control & 33 & 31,26 & 1031,50 & $Z=-1,373$ \\
\hline & Total & 68 & & & Sig. asintota (bilateral) $=, 170$ \\
\hline
\end{tabular}

Fuente: elaboración propia 


\section{Discusión}

Realizada la investigación y en función a los resultados obtenidos en el pretest y postest, se concluye que la educación virtual empleando la plataforma Blackboard influye significativamente en el pensamiento crítico de los estudiantes de una universidad privada de Lima en 2020. En consecuencia, la educación virtual es una metodología a distancia eficaz que coadyuva a que el estudiante analice textos con temas controversiales de contextos internacionales, nacionales y locales, e infiera significados, argumente críticamente posturas a favor o en contra, y formule propuestas de solución a estos temas polémicos (Facione, 2007). Además, se meditó extender un balance con los trabajos ejecutados por otros indagadores, estimando las discusiones a continuación.

Con referencia a la hipótesis general, en la prueba de pretest del pensamiento crítico se visualizó que el grupo experimental alcanzó ciertos resultados en los cuatro niveles en sus calificaciones. Sobre un puntaje de 20, la nota mínima fue de 7 y la máxima de 15; no obstante, después de aplicar el programa sobre educación virtual, con una duración de 12 sesiones, se tomó el postest y se obtuvieron notorios resultados en la mayoría de las dimensiones del pensamiento crítico, como se puede apreciar en el resultado del estadístico ( $p$-valor $=, 000<, 05$ ). Esto evidencia que los estudiantes desarrollaron algunas dimensiones del pensamiento crítico después de la aplicación del programa de la educación virtual.

En cuanto a los resultados del análisis inferencial de la hipótesis general, se determinó que la comparación del grupo control y el grupo experimental en estudiantes de una universidad privada de Lima en 2020, luego de la manipulación de la variable pensamiento crítico empleando la plataforma Blackboard, obtuvo un resultado favorable después de la prueba $U$ de Mann-Whitney, con $p$-valor $=, 000<, 05$. En este sentido, se afirma que la educación virtual influye significativamente en el pensamiento crítico de los estudiantes. Al respecto, experiencias internacionales y nacionales respaldan los resultados, como las de Tabares, Betancourth y Martínez (2019), quienes analizaron un estudio cuasiexperimental, para determinar los efectos de un programa de intervención, basado en debate crítico sobre el pensamiento crítico de estudiantes universitarios. Después de la manipulación de la variable se probó una mejora significativa en el pensamiento crítico, en cuanto a la toma de decisiones, solución de problemas y puntuación total de la prueba. Asimismo, Álvarez (2020), en su estudio de diseño cuasiexperimental, probó el efecto del debate académico en el pensamiento crítico de los estudiantes, y mostró resultados estadísticos significativos entre el grupo de control y el grupo experimental. Al respecto, se establece que la comparación de resultados obtenidos 
concluyó que el debate influye significativamente en el pensamiento crítico. Estos dos estudios demuestran que la implementación de un programa innovador como una actividad pedagógica activa ayuda a desarrollar el pensamiento crítico en los estudiantes.

El presente estudio sobre la educación virtual se respalda en la teoría de Siemens (2004), cuando menciona sobre las conexiones que pueden ser de cualquier ente que desarrollen un aprendizaje digital. De la misma manera, en su teoría sobre la educación virtual Gros (2011) define a la educación virtual como una metodología a distancia que utiliza elementos digitales cuya inclinación va en incremento en relación con su implementación en el contexto. La considera una metodología educativa a distancia cuyo diseño debe estar direccionado según las necesidades de los discentes, con el fin de obtener las habilidades y capacidades previstas. Asimismo, debe contar con tres elementos fundamentales: los recursos de aprendizaje, el soporte técnico virtual y el acompañamiento constante del docente para que el estudiante no se sienta desamparado. Estas ideas son respaldadas por Pando (2018), cuando menciona que el efecto de las TIC mejora el trabajo educativo y sugiere plantear una didáctica crítica como una óptica de reflexión sobre una pedagogía del entorno digital. La interrelación del docente tutor virtual y el estudiante a través de la educación virtual y sus herramientas digitales deben ser permanentes tanto en las actividades síncronas como asíncronas. Entre estas actividades de interrelación se debe realizar la retroalimentación con una comunicación asertiva, empática, clara y precisa. Estas aseveraciones son respaldadas por la teoría sociocultural de Vygotsky, cuyo fundamento es la relación del hombre con sus semejantes para el desarrollo del proceso cognitivo personal. Por último, Rodríguez y Barragán (2017) mencionaron que el uso de los recursos digitales en los docentes y estudiantes aumenta las competencias profesionales en la educación superior. Califica el perfil de la universidad como moderna e innovadora, lo que admite un desarrollo integral del aprendizaje autónomo y constante, al desplegar el progreso digital e involucrar el pensamiento crítico en el momento de asimilar conocimientos.

\section{Contribución de autoría}

Carlos Arturo Valencia Morocho es el autor del artículo y responsable de la estructura y redacción del documento.

\section{Fuente de financiamiento}

Autofinanciado.

\section{Potenciales conflictos de interés}

Ninguno. 


\section{REFERENCIAS BIBLIOGRÁFICAS}

Ahumada, O. y De la Hoz, N. (2019). Mooc y su efecto en el desarrollo del pensamiento crítico de los estudiantes de la básica secundaria en el área de Lengua Castellana. [Tesis de magíster, Universidad de la Costa, Barranquila]. shorturl.at/EJLV6

Álvarez, M. (2020). Efecto del debate académico en el pensamiento crítico en estudiantes de educación secundaria del distrito de San Miguel, año 2019. [Tesis de doctorado, Universidad César Vallejo]. https://repositorio.ucv.edu.pe/bitstream/handle/20.500.12692/42829/Alvarez_HMY. pdf? sequence $=1$ \&isAllowed $=y$

Alves, P., Miranda, L. y Morais, C. (2017). La influencia de los entornos virtuales de aprendizaje en el rendimiento de los estudiantes. Revista Universal de Investigación Educativa, 5(3), 517-527. doi: 10.13189 / ujer.2017.050325. http://www.hrpub.org/journals/article_info.php?aid=5705

Ardoin, M., Clark, C. y Kelsey, E. (2013). An exploration of future trends in environmental education research. Environmental Education Research, 19(4), 499-520. http://dx.doi.org/10.1080/13504622.2012.709823

Campbell, D. y Stanley, J. (1995). Diseño experimentales y cuasiexperimentales en la investigación social. Amorrortu Editores.

Carrasco, S. (2017). Metodología de la investigación científica (8. a edición). San Marcos.

Cuadrao, L. (2016). El método de enseñanza virtual y su influencia en el aprendizaje de histopatología. [Tesis de doctorado, Universidad Nacional Mayor de San Marcos]. http://cybertesis.unmsm.edu.pe/bitstream/handle/cybertesis/5044/Cuadrao_zl.pdf?sequence=1\&isAllow.

Facione, P. (2007). Pensamiento crítico: ¿qué es y porque es importante? Insight Assessment, 1-22. http://eduteka.icesi.edu.co/pdfdir/PensamientoCriticoFacione.pdf

Fierro, M. (2011, setiembre). El desarrollo conceptual de la ciencia cognitiva. Parte I. Revista Colombiana de Psiquiatría, 40(3), 519-533. https://www. redalyc.org/pdf/806/80622315011.pdf

Gros, B. (2011). Evolución y retos de la educación virtual. Construyendo el e-learning del siglo XXI. Editorial UOC. http://redined.mecd. gob.es/xmlui/bitstream/handle/11162/66735/00820122016579. pdf? sequence $=1$ \&isAllowed $=y$

Halmin, L. y Mokhtar, L. (2015, noviembre). Critical thinking process in science learning. Prosiding Seminar Nasional Pendidikan Sains (SNPS) 2015. https://www.researchgate.net/publication/304013631_Critical_ Thinking_Process_in_S cience_Learning 
Hernández, R. y Mendoza, C. (2018). Metodología de la investigación. Las rutas cuantitativas, cualitativas y mixtas. McGraw-Hill Education. http://virtual.cuautitlan.unam.mx/rudics/?p=2612

Huapaya, J. (2016). La educación virtual como modelo didáctico para mejorar la formación profesional de los estudiantes de ingeniería de sistemas de las universidades de la región norte del Perú. [Tesis de doctorado, Universidad Privada Antenor Orrego]. http://repositorio.upao.edu.pe/handle/ upaorep/3681

López, P. (2013). Población, nuestra y muestreo. Punto Cero, 9(8), 69-74. http://www.scielo.org.bo/scielo.php?script=sci_arttext\&pid $=$ S1815-02762004000100012

López, J. (2019). La plataforma virtual Blackboard y el pensamiento crítico en el curso de Análisis de Circuitos Eléctricos, de las carreras de Ingeniería Electrónica e Ingeniería Mecatrónica de la Universidad Peruana de Ciencias Aplicadas en el 2017. [Tesis de maestría, Universidad Nacional de Educación Enrique Guzmán y Valle]. http://repositorio.une.edu.pe/handle/UNE/2936

Ministerio de Educación (2018). Guía de Información Censal de Estudiantes Información para directores y docentes.

OCDE (2016). PISA 2015. Resultados claves. https://www.oecd.org/pisa/ pisa2015-results-in-focus-ESP.pdf

Pando, V. (2018). Tendencias didácticas de la educación virtual: Un enfoque interpretativo. Propósitos y Representaciones, 6(1), 463-505. http:// dx.doi.org/10.20511/pyr2018.v6n1.167

Prada, L. (2016). Influencia de la plataforma «Más Tecnología» sobre el pensamiento crítico. Revista de Innovación Educativa Apertura, 7(2). http://www.udgvirtual.udg.mx/apertura/index.php/apertura/article/ view/696/475

Pérez, L. y López, C. (2008). Técnicas de análisis multivariante de datos, aplicaciones con SPSS. En C. P. López, Introducción a las técnicas de análisis multivariante de datos. Pearson Educación.

Quezada, N. (2015). Métodología de la investigación. Macro.

Rodríguez, M. y Barragán, H. (2017). Entornos virtuales de aprendizaje como apoyo a la enseñanza presencial para potenciar el proceso educativo. Revista Killkana Social, 1(2). doi.org/10.26871/killkana social. v1i2.29

Rojas, E. (2019). Programa didáctico para mejorar el pensamiento crítico en los estudiantes de la especialidad de Lengua y Literatura del segundo ciclo de la Escuela Profesional de Educación Fachse-Unprg, Lambayeque, 2018. [Tesis de maestría en Ciencias de la Educación, Universidad Nacional Pedro Ruiz Gallo]. http://repositorio.unprg.edu.pe/bitstream/handle/UNPRG/3994/ BC-TES-TMP-2800.pdf?sequence=1\&isAllowed=y 
Samaja, J. (2012). Epistemología y metodología. Eudeba. https://www.eudeba.com.ar/Papel/9789502309316/Epistemolog\%C3\%ADa+y+metodol og\%C3\%ADa

Sánchez, H. y Reyes, C. (2015). Metodología y diseños en la investigación científica. (5. a edición). Business Support Aneth. http://www.urp.edu.pe/ pdf/psicologia/publicacion-02.pdf

Santos, C. (2015). Aulas virtuales y foros: Escenarios educativos del siglo XXI. Universidad Autónoma de Bucaramanga. http://190.144.180.114/ handle/11619/3886?show=full

Siemens, G. (2004). Conectivismo: Una teoría de aprendizaje para la era digital. Revista Creative Commons 2.5. https://www.comenius.cl/recursos/ virtual/minsal_v2/Modulo_1/Recursos/Lectura/conectivismo_Siemens. pdf

Stromquist, N. P. y Monkman, K. (Eds.). (2014). Globalization and education: Integration and contestation across cultures. R\&L Education.

Tabares, Y., Betancourth, S. y Martínez, V. (2019, junio). Evaluación del pensamiento crítico en estudiantes de Trabajo Social de la región de Atacama, Chile. Entramado, 16(1), 152-164. https://revistas.unilibre.edu.co/index. php/entramado/article/view/6139/5656.

Unesco (2013). Enfoques estratégicos sobre las TICS en Educación en América Latina y el Caribe. OREAL /Unesco. http://www.unesco.org/new/fileadmin/MULTIMEDIA/FIELD/Santiago/images/ticsesp.pdf

Valderrama, S. (2015). Pasos para elaborar proyectos de investigación científica. San Marcos. https://es.scribd.com/document/335731707/Pasos-Para-Elaborar-Proyectos-de-Investigacion-Cientifica-Santiago-ValderramaMendoza.

Vargas, Z. (2014). La investigación aplicada: una forma de conocer las realidades con evidencia científica. Educación, 38(1), 155-165. https://www. redalyc.org/pdf/440/44015082010.pdf

Vygotsky, L. (1997). The instrumental method in psychology. The collected works of L. S. Vygotsky. Vol. 3. Problems of the theory and history of psychology (pp. 85-89). Plenum Press. 